\title{
вмJ Global Health The case for a global focus on multiple chronic conditions
}

To cite: Hajat C, Kishore SP. The case for a global focus on multiple chronic conditions. BMJ Glob Health 2018;3:e000874. doi:10.1136/ bmjgh-2018-000874

Handling editor Seye Abimbola

Received 9 April 2018 Revised 21 May 2018 Accepted 24 May 2018

\section{Sinked}

- http://dx.doi.org/10.1136/ bmjgh-2017-000505

Check for updates

${ }^{1}$ Public Health Institute, UAE University, Al Ain, Abu Dhabi, United Arab Emirates ${ }^{2}$ Arnhold Institute for Global Health, Icahn School of Medicine, Mount Sinai Health System, New York City, New York, USA

Correspondence to Dr Cother Hajat; chajat@hotmail.com

\section{INTRODUCTION}

One in three adults globally suffers from multiple chronic conditions (MCC). ${ }^{1}$ This figure is closer to three out of four in older adults living in developed countries ${ }^{1}$ and is predicted to rise dramatically, with the proportion of patients with four or more diseases almost doubling between 2015 and 2035 in the UK. ${ }^{2}$

Yet the response from the health community is non-commensurate. In our 2017 overview of the burden from MCCs, presented at a World Economic Forum event in September 2017 alongside the United Nations General Assembly, we concluded that despite MCCs being so prevalent, there is a dearth of information on their prevalence, clustering, cost and patient burden. ${ }^{3}$

A 2018 systematic review published in $B M J$ Global Health by Sum et al aimed to quantify out-of-pocket expenditure (OOPE) on medicines in patients with MCCs. ${ }^{4}$ Using the context of the findings from Sum et al, we argue why MCCs warrant specific focus by the health community.

\section{TERMINOLOGY AND DEFINITIONS}

Challenges persist in framing the topic of MCCs. First, there is no agreed taxonomy. Our research found that several terms including MCCs, multimorbidity, comorbidity and polychronic diseases are all used interchangeably to mean 'more than one chronic condition'. ${ }^{3}$ However, experts deemed multimorbidity to signify end-stage disease and comorbidity to signify only one main condition. ${ }^{3}$ Second, there is no agreed definition of which 'chronic' conditions should be included in the context of MCCs, with studies including between 4 and 147 conditions. ${ }^{3}$ We previously found this lack of consensus can lead to variation in prevalence rates by up to threefold, ${ }^{3}$ and in the review by Sum et al resulted in too much heterogeneity to perform a meta-analysis. ${ }^{4}$ Also of note, the

\section{Summary box}

Consensus agreement on the taxonomy of patients living with more than one chronic condition is needed.

- Prevention of chronic diseases through improvements to lifestyle behaviours remains paramount to tackling multiple chronic conditions (MCC); evaluations to establish cost-effective interventions are required.

- Data reporting and monitoring systems, such as hospital coding, should specifically address and monitor MCCs.

- Healthcare delivery systems need to adapt to manage more than one chronic condition simultaneously; this will be facilitated by innovative changes in healthcare payment mechanisms.

- An increase in the resources to further the evidence base must be part of any strategic solution for MCCs.

review by Sum et al focused on MCC but did not include the broader definition including long-term communicable conditions such as tuberculosis (TB) and HIV/AIDS. ${ }^{56}$

\section{CURRENT GAPS IN THE EVIDENCE BASE}

In addition to the noted dearth of academic literature, ${ }^{3}$ commonly used health, epidemiological and administrative data sources such as WHO, Global Burden of Disease and the International Classification of Diseases coding systems do not report specifically on MCCs which would otherwise contribute significantly to the available data. ${ }^{3}$ The evidence base is particularly scarce in younger populations and low/middle-income country settings. It is worth noting that development assistance for non-communicable diseases (NCD) has historically been disproportionately low: NCDs accounted for $49.8 \%$ of the disease burden whereas the level of health assistance allocated was just $2.3 \%$ to NCDs for low/middle-income countries in $2010 .{ }^{3}$ The amount of funding for MCCs is unknown but is likely to represent only a small proportion of that for NCDs overall. We are unlikely to 
see a change in how chronic conditions are managed until we increase funding for them.

\section{FINANCIAL AND SOCIAL BURDEN}

MCCs accrue a markedly elevated cost and personal burden with some evidence of a doubling of healthcare expenditure with each additional chronic condition. ${ }^{7}$ In addition, individual patients are also impacted by elevated costs of MCCs if they are responsible for expenses associated with their healthcare usage. For example, OOPE is 2.1 times higher for older patients with MCCs than those without MCCs. ${ }^{8}$ Sum et al report that the greater the number of conditions, the higher the OOPE on medicines as a proportion of total healthcare expenditure. ${ }^{4}$ Furthermore, OOPEs for people with MCCs are increasing at a faster rate than wage growth or broader consumer inflation levels ${ }^{4}$ and elderly and low-income families are disproportionately affected. ${ }^{4}$ MCCs have the potential to adversely affect the lives of the most vulnerable members of society, particularly those without adequate health insurance coverage or access to universal healthcare systems. ${ }^{7}$

It is difficult to quantify OOPE accurately due to the sheer complexity of healthcare payment systems, including: large variations in copays, coinsurance, absolute caps and other payment models. As the studies arise from several differing health systems, the variation is likely to be even greater. Nevertheless, as the authors rightly emphasise, this finding highlights an urgent need to review and revise existing OOPE payment requirements for chronic conditions to move from single-disease frameworks to ones that take MCCs into account. Finally, the burden of MCCs also increases the burden of care on individuals while also reducing patients' abilities to care for themselves.

\section{CLUSTERS OF CHRONIC CONDITIONS}

Certain conditions occur together more frequently by virtue of high prevalence rates, shared risk factors or due to causation of one condition by another. A cluster of chronic conditions is when two or more chronic conditions occur together more often than expected.

Common clusters of conditions are shown in table 1 : strong associations exist between depression associated with stroke and Alzheimer's disease, and communicable conditions such as TB and HIV/AIDS with diabetes and cardiovascular disease (CVD), respectively.

Sum et al reported that some clusters of conditions yielded greater OOPE for medications. ${ }^{4}$ This aligns with previous findings of greater healthcare expenditures on specific clusters of conditions. ${ }^{3}$ However, only one of the 14 studies that were included investigated this issue and it was limited to only two clusters of conditions, namely those including diabetes and acute coronary syndrome. While this issue warrants considerable further investigation, caution must be made in the interpretation of the findings from a single study from Australia ${ }^{9}$ in terms of

\begin{tabular}{|c|c|c|}
\hline Condition 1 & Condition 2 & Association* \\
\hline Ischaemic stroke & Depression & Relative risk: $3.19^{1}$ \\
\hline Diabetes & Tuberculosis & OR: $3.11^{5}$ \\
\hline COPD & Tuberculosis & OR: $2.47^{11}$ \\
\hline HIV/AIDS & CVD & $\begin{array}{l}\text { OR }^{6}: \\
\text { On ART: } 2.0 \\
\text { Not on ART: } 1.6\end{array}$ \\
\hline Diabetes & Ischaemic stroke & Relative risk: $1.9^{12}$ \\
\hline Depression & $\begin{array}{l}\text { Alzheimer's and } \\
\text { other dementias }\end{array}$ & Relative risk: $1.85^{1}$ \\
\hline
\end{tabular}

*OR or relative risk (RR) of having condition 2 for those with condition 1.

ART, antiretroviral therapy; COPD, chronic obstructive pulmonary disease; CVD, cardiovascular disease (includes ischaemic stroke and ischaemic heart disease).

making policy recommendations. A thorough investigation of all chronic conditions, including both NCDs and long-term communicable conditions, and covering different geographical health systems, would be required to truly identify which clusters of conditions account for higher OOPE.

In low/middle-income countries, where conditions such as diabetes and TB are highly prevalent, the failure to take clustering into account risks serious failures in the epidemiological estimates and clinical management of these conditions. Clusters are also highly amenable to large improvements in health and cost outcomes through relatively simple shifts in healthcare delivery such as the use of joint disease guidelines that tackle more than one common condition in a cluster, tailored screening and prevention. Healthcare payment mechanisms in developed countries often reward activity rather than desirable outcomes; shifting towards payment for quality or outcomes would facilitate better management of MCCs. As the majority of chronic conditions that are part of MCC clusters are amenable to prevention, interventions that tackle both primary and secondary prevention must be part of the longer term strategy to reducing the MCC burden.

\section{RECOMMENDATIONS}

Key recommendations for moving forward with the science and interventions for MCCs include:

1. Consensus agreement on the taxonomy of MCCs is needed including the terminology, the definition and even what constitutes a chronic condition.

2. Prevention of chronic diseases through improvements to lifestyle behaviours remains paramount to tackling MCCs and economic evaluations to establish the most cost-effective interventions to reduce the MCC burden are required.

3. Data reporting and monitoring systems should specifically address and monitor MCCs; the ability to report 
on multiple primary diagnoses in future coding systems should be considered.

4. Healthcare delivery systems need to adapt to move away from single-disease frameworks to managing more than one chronic condition simultaneously, examples of which are emerging for HIV and CVD management.

5. Healthcare payment mechanisms that reward positive health outcomes rather than activity-based funding may help in achieving improved patient outcomes, such as the shift to outcome-based reimbursement in the USA.

\section{CONCLUSION}

The authors agree with the recommendations of Sum et al that urgent reforms are required in the healthcare delivery and financing mechanisms applied to the management of chronic conditions to move away from a single-disease framework towards one that considers clusters of chronic conditions. By tackling clusters rather than single conditions, interventions and systems can directly address particular difficulties faced by such patients, through medication design (eg, fixed-dose combination pills), approaches for screening and detection (eg, mental health screening in diabetes clinics) and joint care guidelines (eg, for diabetes and TB). These relatively small changes in the way that such care is delivered may lead to considerable changes in the ability to deliver greater patient-centric care. However, the current gaps in evidence base related to MCCs prevent such policy changes being fully informed. An increase in the resources to further the evidence base must be part of any strategic solution for MCCs. The rapid ageing of populations globally and the alarming predictions of increased MCCs render these changes urgent.

Contributors The content of this article is solely that of the two authors. $\mathrm{CH}$ prepared the first draft, SPK contributed to the manuscript.

Competing interests $\mathrm{CH}$ has received support from Teva Pharmaceuticals for analysis on multiple chronic conditions. SPK leads a partnership with Arnhold Institute for Global Health at the Icahn School of Medicine at Mount Sinai and Teva Pharmaceuticals on multiple chronic conditions.
Patient consent Not required.

Provenance and peer review Not commissioned; externally peer reviewed.

Data sharing statement № additional data are available.

Open access This is an open access article distributed in accordance with the Creative Commons Attribution Non Commercial (CC BY-NC 4.0) license, which permits others to distribute, remix, adapt, build upon this work non-commercially, and license their derivative works on different terms, provided the original work is properly cited and the use is non-commercial. See: http://creativecommons.org/ licenses/by-nc/4.0/

(c) Article author(s) (or their employer(s) unless otherwise stated in the text of the article) 2018. All rights reserved. No commercial use is permitted unless otherwise expressly granted.

\section{REFERENCES}

1. Marengoni A, Angleman S, Melis R, et al. Aging with multimorbidity: a systematic review of the literature. Ageing Res Rev 2011;10:430-9.

2. Kingston $\mathrm{A}$, Robinson $\mathrm{L}$, Booth $\mathrm{H}$, et al. Projections of multimorbidity in the older population in England to 2035: estimates from the Population Ageing and Care Simulation (PACSim) model. Age Ageing 2018;47:374-80.

3. Hajat C, Stein E, Yach D. Multiple chronic conditions: the global state. https://Inkd.in/etb9eAJ

4. Sum G, Hone T, Atun R, et al. Multimorbidity and out-of-pocket expenditure on medicines: a systematic review. BMJ Glob Health 2018;3:e000505.

5. Jeon CY, Murray MB. Diabetes mellitus increases the risk of active tuberculosis: a systematic review of 13 observational studies. PLOS Med 2008;5:e152.

6. Islam FM, Wu J, Jansson J, et al. Relative risk of cardiovascular disease among people living with HIV: a systematic review and meta-analysis. HIV Med 2012;13:453-68.

7. McPhail SM. Multimorbidity in chronic disease: impact on health care resources and costs. Risk Manag Healthc Policy 2016;9:143-56.

8. Paez KA, Zhao L, Hwang W. Rising out-of-pocket spending for chronic conditions: a ten-year trend. Health Aff 2009;28:15-25.

9. Kemp A, Preen DB, Glover J, et al. Impact of cost of medicines for chronic conditions on low income households in Australia. $J$ Health Serv Res Policy 2013;18:21-7.

10. Huang CQ, Dong BR, Lu ZC, Z-c L, et al. Chronic diseases and risk for depression in old age: a meta-analysis of published literature. Ageing Res Rev 2010;9:131-41.

11. Lee $\mathrm{CH}$, Lee MC, Shu CC, et al. Risk factors for pulmonary tuberculosis in patients with chronic obstructive airway disease in Taiwan: a nationwide cohort study. BMC Infect Dis 2013;13:194.

12. Barrett-Connor E, Khaw KT. Diabetes mellitus: an independent risk factor for stroke? Am J Epidemiol 1988;128:116-23.

13. Tatemichi TK, Paik M, Bagiella E, et al. Risk of dementia after stroke in a hospitalized cohort: results of a longitudinal study. Neurology 1994;44:1885-91. 